\title{
The Potsdam contribution to the extragalactic link of the Hipparcos proper motion system
}

\author{
S. Hirte ${ }^{1}$, E. Schilbach ${ }^{1}$, and R.-D. Scholz ${ }^{1}$ \\ WIP Astronomie, Universität Potsdam, An der Sternwarte 16, D-14482 Potsdam, Germany
}

Received January 13; accepted February 17, 1997

\begin{abstract}
Absolute proper motions of 360 Hipparcos stars in 24 fields well distributed over the northern sky were derived from measurements of photographic plates taken mainly with the Tautenburg Schmidt telescope $(134 / 200 / 400 \mathrm{~cm})$. In each field (about 10 square degrees) a large number of galaxies was used to link the proper motions to the extragalactic reference system. Because of systematic magnitude-dependent errors, which affect the proper motion determination of bright stars, only the 256 stars with $B \geq 9.0$ were used to determine the spin parameters of the Hipparcos system. The accuracy of the final result is $0.5 \mathrm{mas} / \mathrm{yr}$ for all three spin parameters.
\end{abstract}

Key words: astrometry - methods: statistical reference systems — catalogs — stars: kinematics

\section{Introduction}

The ESA satellite Hipparcos has measured positions, proper motions and parallaxes of more than 100000 stars in a homogeneous system with a very high internal accuracy. Nevertheless, the Hipparcos reference frame needed a link with regard to an inertial system. There are different methods to link the Hipparcos reference frame to extragalactic objects (Lindegren \& Kovalevsky 1995). One possibility is to use photographic astrometry with deep Schmidt plates for this link.

In 1986 the Potsdam group started its programme to link the Hipparcos proper motion system to an extragalactic reference system using the Tautenburg Schmidt telescope. First investigations showed that Tautenburg plates provide proper motions with respect to extragalactic objects with an accuracy of about 6 mas/yr for bright stars with $B \sim 9$ (Schilbach 1984; Scholz 1991). A first rough estimation showed that a theoretical accuracy of 1.2 mas/yr for the transformation of the Hipparcos proper motion system can be achieved with six well-distributed link fields

Send offprint requests to: S. Hirte
(Dick et al. 1987). Numerical simulations carried out with 10 fields and 119 link stars gave an accuracy of 1.4 mas/yr (Yatsenko et al. 1987). Later reports on the status of the Potsdam programme for the Hipparcos extragalactic link (Brosche et al. 1992, 1995) confirmed the more optimistic expectation of accuracies better than 1.0 mas/yr. Special efforts were made in order to increase the number of link fields.

A check of the Tautenburg plate archive showed that there are more than 70 fields with at least 4 plates taken between 1960 and 1970 which could be used as first epoch plates for the determination of proper motions (Scholz \& Hirte 1991). Altogether, 26 fields (see Fig. 1) included in different Potsdam proper motion programmes were selected for the Hipparcos link.

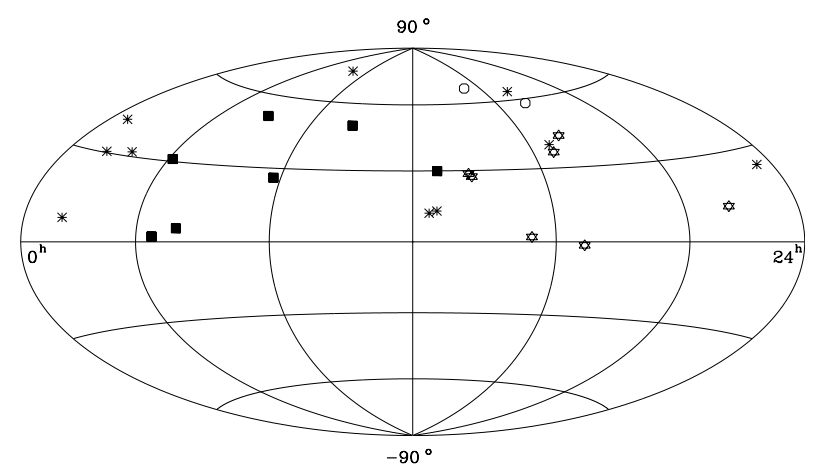

Fig. 1. Distribution of the link fields in equatorial coordinates. Filled squares: MEGA fields, stars: globular cluster fields, open circles: fields with dwarf spheroidal satellites of the Galaxy (excluded from final solution), asterisks: other fields

\section{Observations and measurements}

For the Potsdam programme of linking the proper motions of the provisional Hipparcos catalogue H37cr to an extragalactic reference system we used exclusively deep 
Table 1. Link fields

\begin{tabular}{|c|c|c|c|c|c|c|c|c|c|}
\hline field & $\begin{array}{l}\text { R.A. } \\
\text { J19؛ }\end{array}$ & Dec. & $\begin{array}{c}\text { number of } \\
\text { plates }\end{array}$ & $\begin{array}{c}\text { measuring } \\
\text { device }\end{array}$ & $\begin{array}{l}\text { baseline } \\
\text { [years] }\end{array}$ & $\begin{array}{c}\text { number of } \\
\text { galaxies }\end{array}$ & $\begin{array}{c}\text { all } \\
\text { Hipparcos } \\
\text { stars }\end{array}$ & $\begin{array}{c}\text { reliably } \\
\text { measured } \\
\text { H37cr stars }\end{array}$ & $\begin{array}{c}\text { finally } \\
\text { used stars } \\
(B \geq 9)\end{array}$ \\
\hline M31 & 10.7 & 40.9 & $8 \mathrm{~T}$ & PARSEK & 25 & 70 & 36 & 32 & 20 \\
\hline A193 & 21.4 & 7.9 & $4 \mathrm{~T}$ & PDS & 21 & 150 & 23 & 13 & 11 \\
\hline M33 & 23.1 & 30.4 & $4 \mathrm{~T}$ & ASCORECORD & 18 & 60 & 26 & 23 & 10 \\
\hline SNHoffm & 37.2 & 31.6 & $4 \mathrm{~T}$ & PDS & 29 & 30 & 23 & 15 & 4 \\
\hline MEGA01 & 62.0 & 30.9 & $4 \mathrm{~T}$ & MAMA & 25 & 270 & 23 & 10 & 7 \\
\hline MEGA02 & 67.5 & 2.0 & $4 \mathrm{~T}$ & MAMA & 19 & 120 & 22 & 12 & 11 \\
\hline MEGA04 & 78.5 & 5.1 & $4 \mathrm{~T}$ & MAMA & 25 & 70 & 33 & 20 & 10 \\
\hline MEGA07 & 90.0 & 51.3 & $4 \mathrm{~T}$ & MAMA & 25 & 130 & 30 & 20 & 14 \\
\hline Mrk4 & 100.4 & 74.9 & $4 \mathrm{~T}$ & PARSEK & 22 & 100 & 33 & 17 & 10 \\
\hline MEGA10 & 116.6 & 26.1 & $4 \mathrm{~T}$ & MAMA & 24 & 30 & 15 & 10 & 5 \\
\hline MEGA11 & 144.9 & 49.7 & $4 \mathrm{~T}$ & MAMA & 19 & 190 & 32 & 15 & 13 \\
\hline Virgo2 & 186.9 & 12.0 & $4 \mathrm{~T}$ & APM & 25 & 540 & 21 & 11 & 8 \\
\hline Virgo3 & 190.2 & 12.9 & $4 \mathrm{~T}$ & APM & 24 & 680 & 32 & 10 & 10 \\
\hline MEGA14 & 191.3 & 29.9 & $4 \mathrm{~T}$ & MAMA & 25 & 600 & 30 & 18 & 14 \\
\hline M3-Z & 205.5 & 28.6 & $53 \mathrm{~T}$ & APM & 30 & 1600 & 34 & 14 & 14 \\
\hline M3-IV & 206.8 & 27.4 & $4 \mathrm{~T}$ & APM & 22 & 1050 & 32 & 6 & 6 \\
\hline UMi & 226.4 & 67.2 & $4 \mathrm{P}, 3 \mathrm{~T}$ & APM & 35 & 1280 & 24 & 0 & 0 \\
\hline M5 & 229.7 & 2.0 & $2 \mathrm{P}, 1 \mathrm{UK}, 5 \mathrm{~T}$ & APM & 38 & 1160 & 38 & 15 & 14 \\
\hline M13 & 250.4 & 36.6 & $4 \mathrm{~T}$ & ASCORECORD & 21 & 50 & 36 & 25 & 20 \\
\hline 3C345 & 250.6 & 39.8 & $11 \mathrm{~T}$ & PDS & 20 & 120 & 28 & 17 & 16 \\
\hline M12 & 252.3 & -1.4 & $2 \mathrm{P}, 1 \mathrm{UK}, 2 \mathrm{~T}$ & APM & 43 & 1380 & 24 & 7 & 6 \\
\hline A2255 & 258.8 & 63.9 & $4 \mathrm{~T}$ & PARSEK & 18 & 190 & 25 & 14 & 7 \\
\hline M92 & 259.4 & 43.3 & $10 \mathrm{~T}$ & APM & 26 & 2300 & 27 & 7 & 7 \\
\hline Draco & 260.2 & 58.1 & $4 \mathrm{P}, 3 \mathrm{~T}$ & APM & 37 & 820 & 18 & 0 & 0 \\
\hline M15 & 322.3 & 12.2 & $2 \mathrm{P}, 6 \mathrm{~T}$ & APM & 24 & 510 & 28 & 11 & 11 \\
\hline Mrk319 & 351.2 & 24.4 & $4 \mathrm{~T}$ & PDS & 21 & 50 & 28 & 21 & 11 \\
\hline total & & & & & & & $706^{+}$ & $360^{*}$ & $256^{*}$ \\
\hline
\end{tabular}

T - Tautenburg, P - Palomar, UK - AAO-Schmidt plates.
+ there are 15 common stars in the overlapping field of M3-Z and M3-IV, ${ }^{*} 3$ stars were measured in M3-Z and M3-IV.

Schmidt plates (Table 1). Most of these plates were taken with the Tautenburg Schmidt telescope (134 cm aperture $/ 200 \mathrm{~cm}$ diameter of the mirror $/ 400 \mathrm{~cm}$ focal length). In addition, some plates from the Palomar (122/183/307) and UK (120/180/306) Schmidt telescopes were combined with Tautenburg plates (see Col. 4 of Table 1). However, this combination of plates taken with different Schmidt telescopes and with different plate centres caused problems in the determination of accurate proper motions of the relatively bright Hipparcos link stars (see Sect. 3). For each field, at least two first- and two second-epoch Schmidt plates were used. All plates of the same field were measured with the same measuring device.

Whereas the initial aim was to determine absolute proper motions of Hipparcos stars in 10 Tautenburg proper motion fields (cf. Dick et al. 1987), later on other fields from different Potsdam proper motion projects were included. The final sample of Hipparcos link fields consists of 7 fields from the MEGA programme (Schilbach 1988), 7 fields with globular clusters and 9 other fields with a sufficient number of galaxies as reference points. The results for one field (3C345) were kindly provided by Tucholke (1995) who also used deep Tautenburg plates. Two fields with dwarf spheroidal satellites (dSph) of the Galaxy (Scholz \& Irwin 1994) were later excluded from the link due to large proper motion errors of the Hipparcos stars (see Sect. 3). Altogether, the Hipparcos link fields with absolute proper motions based on deep Schmidt plates are well distributed over the northern sky (see Fig. 1). Each field covers about 10 square degrees. The fields M3-Z (centered on the globular cluster) and M3-IV (with the cluster in the plate corner) overlap by about 25 per cent.

Among all available Hipparcos stars in each field only those with reliable measurements were selected for the proper motion determination (see Fig. 2). The reliability of the measurements was estimated from the image parameters used in the classification of objects (APM, MAMA, PDS) and/or from the internal accuracy of the determined proper motions (comparison of independent results from different plate pairs). The number of the reference galaxies used per field varied in dependence on the Galactic latitude as well as on the measuring and reduction process (see Table 1).

The first three fields included in the Hipparcos extragalactic link programme were M33, M13 and M31 (Scholz 1991). The first two of them had been measured with the semi-automated measuring device ASCORECORD at the Lohrmann-Observatorium of the Technical University Dresden (Schilbach 1984), whereas the M31 field was measured with the automated measuring machine PARSEK at the Main Astronomical Observatory of the Ukrainian Academy of Sciences at Kiev (Sergeev \& Schornikov 1984). 


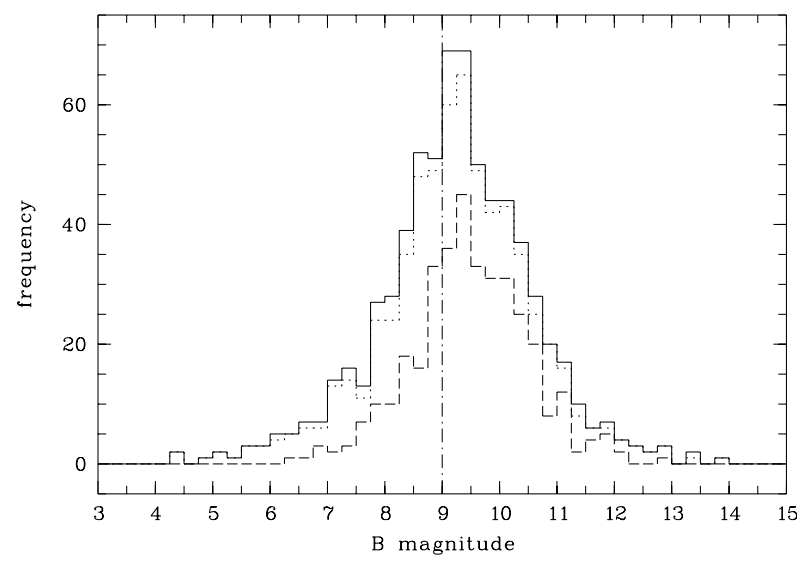

Fig. 2. Number of Hipparcos stars in 26 link fields. Solid line: all available Hipparcos stars, dotted line: stars of the provisional Hipparcos solution H37cr provided for the link, dashed line: selected stars from $\mathrm{H} 37 \mathrm{cr}$ with reliable measurements. Only reliably measured stars with $\mathrm{H} 37 \mathrm{cr}$ data and $B \geq 9$ (on the right side of the dot-dashed line) were used in the final solution

All available Hipparcos stars, but only a limited number of visually classified galaxies could be measured with the ASCORECORD. The same was due for the PARSEK measuring machine which needs rough input coordinates of all objects to be measured. In later PARSEK measurements (fields Mrk 4 and A 2255) we used the image classification on APM-measured POSS1 plates to provide input coordinates of reference galaxies in these two fields. This image classification was visually checked during the automated PARSEK measurements.

For the majority of link fields the Schmidt plates were fully scanned by the automated measuring machines APM (Kibblewhite et al. 1984) at Cambridge, MAMA (Guibert 1983) at Paris and PDS (Horstmann et al. 1989) at Münster. In dependence on the available reduction software, the images and/or image parameters of the Hipparcos stars and of potential reference galaxies were investigated and a selection of well measured objects was made.

So we used standard APM software for the classification of all measured objects into stars, nonstellar objects, noise images and merged objects. In order to exclude those Hipparcos stars which were unreliably measured, only those images classified as stars on all plates were used in the proper motion determination. The reference galaxies were selected according to their classification on the deepest plates. The measurements were corrected for the known periodic errors of the APM (Evans 1988; Evans \& Irwin 1995).

The reduction of the MAMA measurements is described in Kharchenko \& Schilbach (1994). For the clas- sification of the measured objects the MAMA image parameters were used (Schilbach \& Scholz 1991, 1992).

APM and MAMA measurements of the same two plates were used for a comparison of the accuracy of both machines (Schilbach \& Scholz 1992). A somewhat better accuracy for bright stars (in the magnitude range of the Hipparcos catalogue) was found for the MAMA measurements. On the other hand the faint objects (i.e. in the magnitude interval of the reference galaxies) were more accurately measured by the APM.

In the case of PDS measurements we took advantage of the specific output data in the Münster software (Horstmann et al. 1989), i.e. both image parameters and pixel data for each measured object. Due to the special output data format, both automatic image classification and visual check of the measured images are possible. In the proper motion determination we preferred to use those reference galaxies classified by both procedures. Among the measured Hipparcos stars we selected for further reduction only those fitted by a Gaussian profile on all plates.

\section{Determination of absolute proper motions}

For the majority of the link fields only two plate pairs, i.e. two first epoch plates and two second epoch plates were measured. Plate pairs were also used in the fields of M31 (4 pairs), the dSph fields UMi and Draco (3 pairs, for more details, see Scholz \& Irwin 1994) and M92 (5 pairs, see Scholz et al. 1994). For details on the reduction within the MEGA programme, see Schilbach \& Scholz (1991) and Kharchenko et al. (1994).

The proper motion determination in all these fields was based on independent plate-to-plate solutions using polynomials of up to fourth order and the method of stepwise regression of Hirte et al. (1990). The order of the polynomial was chosen according to the number of reference objects (field stars or galaxies) used. Generally, the reduction was done with large numbers of reference galaxies selected from the full plate scans by APM, MAMA and PDS and with third order polynomials. In cases of relatively small numbers of reference galaxies we used field stars in the differential plate-to-plate solutions. The absolute proper motions were then obtained by subtracting the mean "proper motion" of the galaxies per field. Plate pairs measured with ASCORECORD and PARSEK were reduced using the galaxies directly as reference points.

The reduction of the globular cluster fields M5, M12 and M15 is described in Scholz et al. (1996). Here we preferred to use an iterative plate overlap method (Eichhorn 1960) with a catalogue of positions and zero proper motions of the selected reference galaxies as starting point. The plate overlap method was also applied by Tucholke (1995) for the field 3C345. His results based on 3 first and 8 second epoch Tautenburg plates are also included in the present paper. 
A new reduction of the field around the globular cluster M3 (Scholz et al. 1996) was carried out with the aim of a QSO survey based on variability and zero proper motions. Here we have used the proper motions of the Hipparcos stars obtained from 53 blue Tautenburg plates. For this special case of a large number of plates taken over a time baseline of 30 years, we built up a time series of coordinates for each object. The measuring coordinate frame of each plate was transformed to that of the master plate by using a quadratic $2 \times 6$ plate constant polynomial relationship. On each comparison plate the transformed coordinates were shifted by the negative mean coordinate differences of all available galaxies. The absolute proper motions of all objects were then determined from the linear regression of their coordinates over the time.

The absolute stellar proper motions in the 26 Hipparcos link fields were determined with respect to 30 up to 2000 reference galaxies per field (only 7 fields with less than 100 galaxies, see Table 1). With epoch differences of about 20 to 40 years an internal accuracy of 3 to $5 \mathrm{mas} / \mathrm{yr}$ was achieved for undisturbed (by overlapping images) and relatively faint $(B>9)$ Hipparcos stars. Serious problems in the internal proper motion accuracy of bright stars appeared in some of those cases, where we combined plates from different Schmidt telescopes. Especially in the fields of the dSph's Draco and UMi, each with two first epoch POSS1 plates, two second epoch Palomar plates with different plate centre and three third epoch Tautenburg plates, we failed to obtain accurate proper motions of all bright stars $(B<12)$. Therefore, these two fields were excluded from the extragalactic link of the Hipparcos proper motions. In the field of M15 similar problems arised so that we re-reduced the proper motions of the Hipparcos stars in this field without the use of the POSS1 plates.

From 706 Hipparcos stars in all 26 fields H37cr data of $651(92 \%)$ stars were provided for the link. From all measured H37cr stars we excluded those stars which showed large errors in their proper motions because of bad measurements or nonstellar image classification. 360 stars remained for the determination of the link.

Considering the final 24 Hipparcos link fields, the following "success rates" were obtained for the measuring machines used: $34 \%$ of all available Hipparcos stars in 8 fields reliably measured with the APM, $57 \%$ in 7 fields measured with MAMA, $65 \%$ in 4 fields measured with the PDS, $67 \%$ in 3 fields measured with PARSEK and $77 \%$ in 2 fields measured with the ASCORECORD. The smaller "success rate" of the APM in measuring accurate proper motions of Hipparcos stars confirms our results from the comparison of APM and MAMA (Schilbach \& Scholz 1992).

Besides of the random errors arising from the measuring accuracy, all proper motions in a given field are affected by a systematic error which depends on how accurate the extragalactic reference frame was defined by the measurements of the galaxies in that particular field. Due to the large number of galaxies, the formal zero point error in a given field is usually less than 1 mas/yr. However, in a few fields with globular and open clusters systematic magnitude dependent errors in the proper motions were detected and removed (Scholz \& Kharchenko 1994; Kharchenko \& Schilbach 1995). In some of the link fields this kind of error was also found and corrected for. But it can not always be detected so that on the average, residual systematic errors of about 2 mas/yr per field may be expected.

\section{Determination of the spin parameters}

The systematic part of the difference between the Hipparcos positions and positions in an inertial reference system can be described by a rotation. Whereas, the differences in proper motions are represented by the spin, the time derivative of the rotation. For the determination of the spin vector $\omega=\left(\omega_{x}, \omega_{y}, \omega_{z}\right)$ we used the basic equations

$$
\begin{aligned}
& \Delta \mu_{\alpha} \cos \delta=-\omega_{x} \cos \alpha \sin \delta-\omega_{y} \sin \alpha \sin \delta+\omega_{z} \cos \delta \\
& \Delta \mu_{\delta}=+\omega_{x} \sin \alpha \quad-\omega_{y} \cos \alpha \text {. }
\end{aligned}
$$

$\Delta \mu_{\alpha}$ and $\Delta \mu_{\delta}$ are the proper motion differences in the sense Hipparcos minus absolute, $\alpha$ and $\delta$ are the equatorial coordinates of the link stars. The indices $x$ to $z$ refer to a right-handed triad with the $x$-axis pointing towards the vernal equinox and the $z$-axis towards the north equatorial pole.

The $\omega_{x}, \omega_{y}, \omega_{z}$ were computed from both components of the proper motion differences, i.e. from the least squares solution of $2 n$ equations, where $n$ is the number of link stars. This is called the "star solution". For three out of 360 stars used in the solution, proper motions were determined in two fields (M3-Z and M3-IV). In the star solution we used the average of both proper motion determinations for these stars.

The proper motion differences $\Delta \mu_{\alpha} \cos \delta, \Delta \mu_{\delta}$ in a given field may be correlated due to a systematic error of the extragalactic link in each field. Therefore, we used the average proper motion differences for each field in a second least squares solution of $2 m$ equations, where $m$ is the number of link fields. This is called the "field solution".

Both, star and field solution should be identical if the stars are homogeneously distributed in the fields with the same weights and if the fields are homogeneously distributed over the sky. This is not the case, therefore the solutions will differ. Formally, the rms error of the star solution is smaller than that of the field solution because of the greater number of equations.

Using H37cr data and the absolute proper motions of all 360 stars in the whole magnitude interval $6<B<13$ the following results were obtained, for the star solution and the field solution, respectively: 
preliminary solution with 360 stars in 24 fields

referred to the final Hipparcos system (see Sect. 6)

star solution

field solution

residual spin parameters $[\mathrm{mas} / \mathrm{yr}]$

$\begin{array}{ll}\Delta \omega_{x}=+0.59 \pm 0.47 & \Delta \omega_{x}=+0.42 \pm 0.82 \\ \Delta \omega_{y}=+1.08 \pm 0.45 & \Delta \omega_{y}=+0.83 \pm 0.79 \\ \Delta \omega_{z}=+0.18 \pm 0.44 & \Delta \omega_{z}=+0.47 \pm 0.74\end{array}$

$$
\begin{array}{cc}
6.9 \text { mas } / \mathrm{yr} & \text { rms of solution } \\
\text { correlation coefficients }
\end{array}
$$

\section{Investigation of magnitude dependent errors}

Figure 3 and 4 show the proper motion differences H37crabsolute for all 360 selected Hipparcos stars before the determination of the spin parameters. The data were binned in five intervals, with 27, 77, 145, 84 and 26 (from bright to faint) stars, respectively.

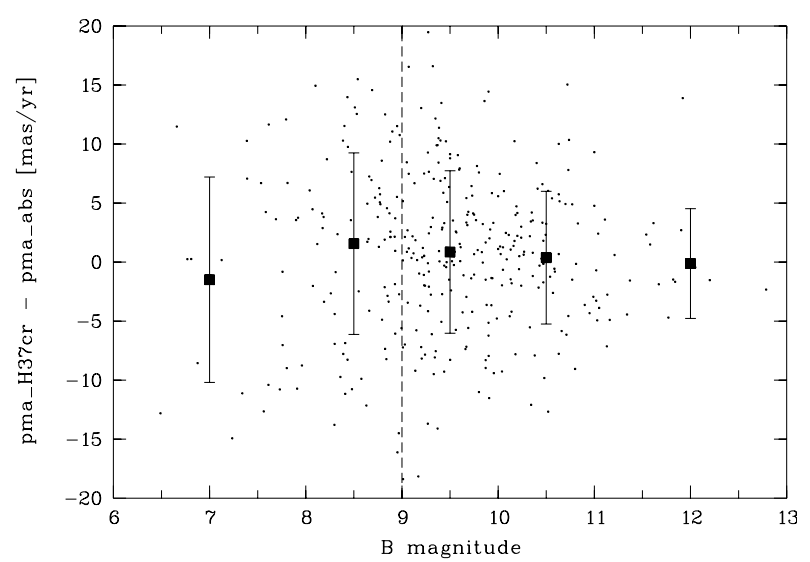

Fig. 3. Proper motion differences $\mathrm{H} 37 \mathrm{cr}-$ absolute in $\mu_{\alpha} \cos \delta$ over the $B$ magnitude. All 360 stars with reliable measurements are plotted. Filled squares with error bars show the mean differences and their dispersions in five intervals: $B<8,8 \leq B<9,9 \leq B<10,10 \leq B<11$ and $B \geq 11$. Only the stars on the right side of the dashed line $(B \geq 9)$ were used in the final solution

Figure 5 shows the dispersion of the proper motion differences $\mathrm{H} 37 \mathrm{cr}$-absolute for the same magnitude intervals. A clear trend towards smaller dispersions with fainter stars can be seen. The larger dispersions of the proper motion differences H37cr-absolute of bright stars in Fig. 5 are caused by larger errors in our proper motion determination for these stars. There is a deviation of the mean differences H37cr-absolute from zero seen in Fig. 3, and especially in Fig. 4 for the bright stars. This possible systematic error in the obtained absolute proper motions of bright stars makes an exclusion of these stars from the fi-

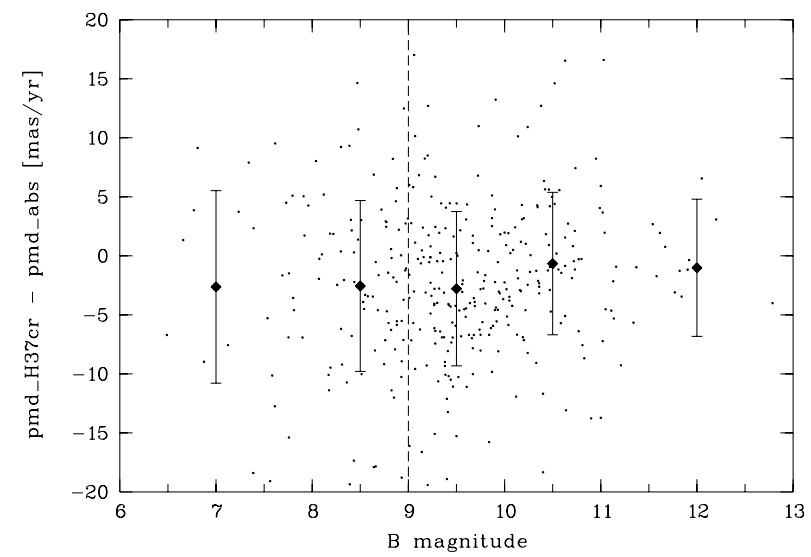

Fig. 4. Same as Fig. 3, but for proper motion differences $\mu_{\delta}$. Filled rhombs and error bars show the mean proper motion differences and their dispersions over the $B$ magnitude

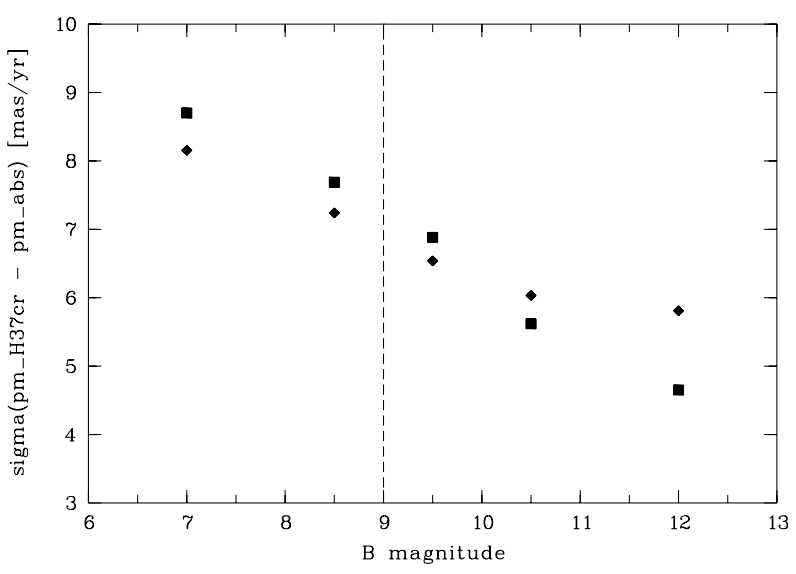

Fig. 5. Change of the dispersion of the proper motion differences $\mathrm{H} 37 \mathrm{cr}-$ absolute in $\mu_{\alpha} \cos \delta$ (filled squares) and $\mu_{\delta}$ (filled rhombs) over the $B$ magnitude. The 360 stars with reliable measurements were binned in five intervals: $B<8,8 \leq B<9,9 \leq B<10,10 \leq B<11$ and $B \geq 11$. Only the stars on the right side of the dashed line $(B \geq 9)$ were used in the final solution

nal link plausible. A further argument for their exclusion is their nonuniform distribution over the link fields (compare the last two columns in Table 1) which may lead to systematic effect in the determination of the spin parameters.

A simple test of the magnitude dependence of the link coefficients was made: The whole set was subdivided into 2 subsets of 180 bright $(B \leq 9.5)$ and 180 faint $(B>9.5)$ stars. The computation of the rotation parameters $\omega_{x}, \omega_{y}$, $\omega_{z}$ yielded significantly different results for these subsets. To study this magnitude dependent effect in more detail, 

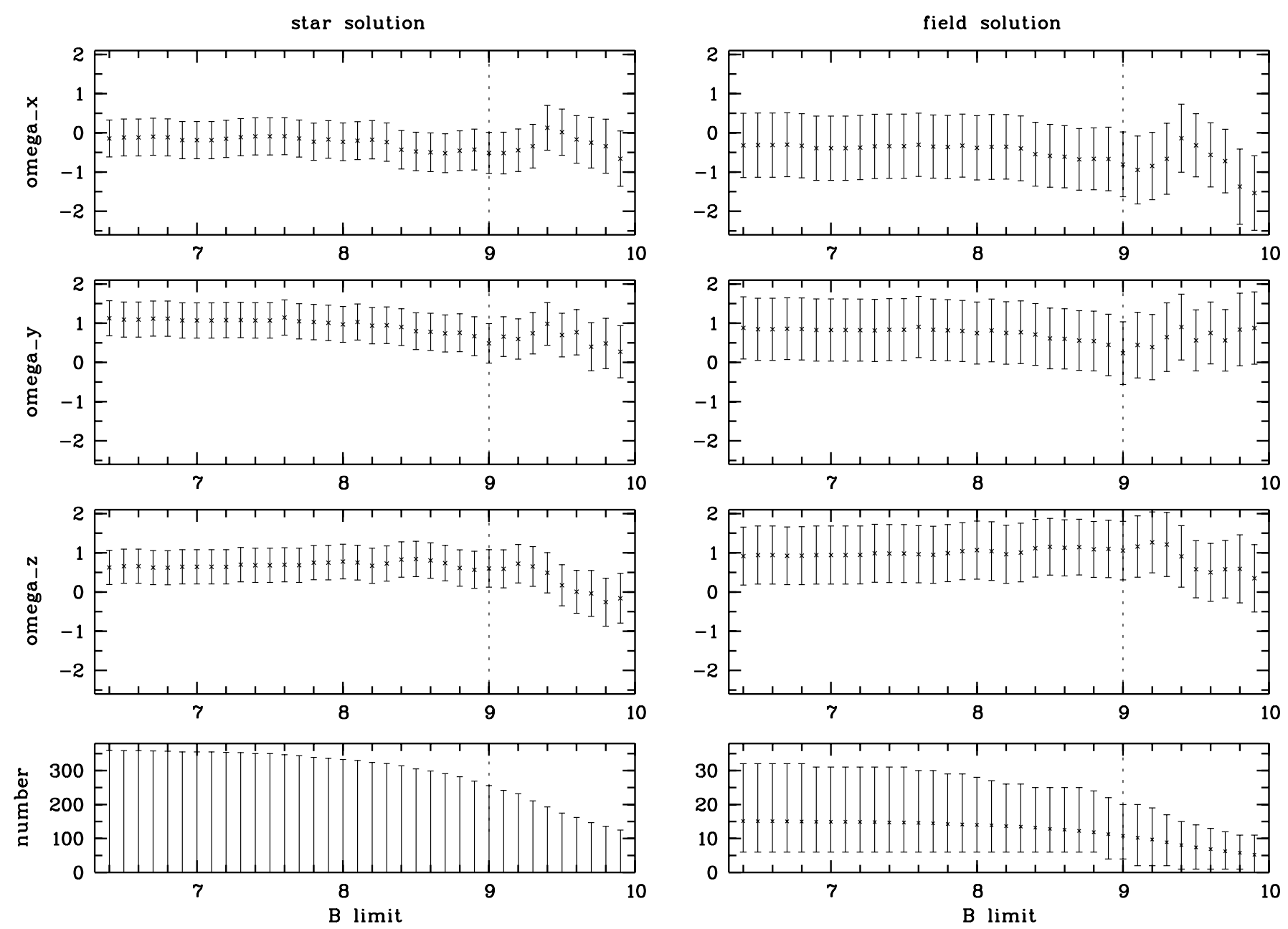

Fig. 6. Values of $\omega$ computed from all stars with $B \geq B_{\text {limit }}$. The lower diagrams show for the star solution the number of stars included and for the field solution the range of the number of stars per field and as crosses the mean number over all fields

the bright stars were successively omitted from the solution in steps of $0.1 \mathrm{mag}$ (see Fig. 6). Due to the relatively small number of bright stars (only 27 stars with $B<8$ ) there are almost no changes in the left part of the diagrams in Fig. 6. But from $B_{\text {limit }}=8$ to $B_{\text {limit }}=9$ a trend can be seen, i.e. the bright stars increase the values of $\omega_{x}$ and $\omega_{y}$ systematically. The number of stars per field (see lower right diagram of Fig. 6) decreases rapidly for $B_{\text {limit }}>9$. From $B_{\text {limit }} \geq 9.4$ there are only one or no stars in some of the link fields, i.e. the solution becomes unstable. Therefore, the selected value $B_{\text {limit }}=9.0$ represents a compromise between a sufficient number of stars (per link field) and a minimum influence of bright stars affected by larger (and possible systematic) proper motion errors.
Final solution with 256 stars in 24 fields referred to the final Hipparcos system (see Sect. 6) star solution field solution residual spin parameters $[\mathrm{mas} / \mathrm{yr}]$

$$
\begin{array}{ll}
\Delta \omega_{x}=-0.22 \pm 0.52 & \Delta \omega_{x}=-0.07 \pm 0.83 \\
\Delta \omega_{y}=+0.43 \pm 0.50 & \Delta \omega_{y}=+0.19 \pm 0.80 \\
\Delta \omega_{z}=+0.13 \pm 0.48 & \Delta \omega_{z}=+0.60 \pm 0.75
\end{array}
$$

$$
\begin{array}{cc}
6.5 \mathrm{mas} / \mathrm{yr} & \text { rms of solution } \\
\text { correlation coefficients } & 3.1 \mathrm{mas} / \mathrm{yr} \\
r_{x y}=+0.20 & r_{x y}=+0.21 \\
r_{x z}=-0.04 & r_{x z}=-0.04 \\
r_{y z}=-0.04 & r_{y z}=-0.01 .
\end{array}
$$

The final solution for the spin parameters was obtained with 256 stars with $B \geq 9.0$ distributed over 24 link fields. The minimum number of stars per field was 4 .

The star solution and the field solution show the same behaviour in Fig. 6 although the errors of the spin parameters from the field solution are larger. In every case, the star solution and the field solution agree within their errors. 


\section{Discussion of the results}

All determinations of the spin parameters were carried out by the use of $\mathrm{H} 37 \mathrm{cr}$ data. In order to express our results in the final Hipparcos system, we compared them with the composed solution of Kovalevsky et al. (1997) based on different programmes and techniques. In this synthesised solution the Potsdam star solution with 256 stars was included.

The residual spin parameters of the Potsdam solution in the final Hipparcos system were obtained by subtracting the composed solution of Kovalevsky et al. (1997)

$$
\left(\omega_{x}, \omega_{y}, \omega_{z}\right)=(-0.73,+0.05,+0.45)[\mathrm{mas} / \mathrm{yr}]
$$

from the Potsdam solution with H37cr. All residual spin parameters $\Delta \omega$ of the final Potsdam solution are smaller than their errors. Table 3 from Kovalevsky et al. (1997) shows the residual spin parameters for the different programmes used in the synthesised solution. The high quality of the Potsdam contribution is underlined by its increased weight in the process of the synthesised solution by Kovalevsky et al. (1997) and the relatively small deviation from the VLBI data. From the comparison with the results presented in Kovalevsky et al. (1997) we may conclude that the small internal errors of the Potsdam spin parameters $(\sim 0.5 \mathrm{mas} / \mathrm{yr})$ correspond to the real errors.

Acknowledgements. We would like to thank the Karl-Schwarzschild-Observatorium of the Thüringer Landessternwarte Tautenburg for supplying the large number of Schmidt plates used for this project. Furthermore, we are grateful for extensive support with the measurements on the APM (IoA Cambridge), MAMA (Observatoire de Paris), PDS (University Münster), PARSEK (Main Astronomical Observatory of Ukrainian Academy of Sciences, Kiev) and ASCORECORD (Technical University Dresden) measuring machines. We thank H.-J. Tucholke for providing his results in the field of 3C345 for this work.

\section{References}

Brosche P., Dick W.R., Galas R., Geffert M., Hirte S., Schilbach E., Scholz R.-D., 1992, Linking HIPPARCOS to galaxies: the Bonn and Potsdam programmes. In: Bergeron J. (ed.) Highlights of Astronomy, Vol. 9, Proc. HIPPARCOS Joint Discussion at XXI. IAU General Assembly. Kluwer Acad. Publ., Dordrecht, p. 439

Brosche P., Geffert M., Hirte S., et al., 1995, HIPPARCOS extragalactic link: preliminary Bonn, Potsdam and Kiev solution. In: Høg E., Seidelman K. (eds.) Proc. IAU Symposium 166, Astronomical and astrophysical objectives of submilliarcsecond optical astrometry. Kluwer Acad. Publ.,
Dordrecht, p. 380

Dick W.R., Ruben G., Schilbach E., Scholz R.-D., 1987, Astron. Nachr. 308, 211

Eichhorn H., 1960, Astron. Nachr. 285, 233

Evans D.W., 1988, PhD Thesis, Cambridge

Evans D.W., Irwin, M., 1995, MNRAS 277, 820

Guibert J., 1983, Bull. Inform. CDS 25, 13

Hirte S., Dick W.R., Schilbach E., Scholz R.-D., 1990, Application of stepwise regression in photographic astrometry. In: Jaschek C., Murtagh F. (eds.) Proc. of Conference on Errors, Uncertainties and Bias in Astronomy. Cambridge Univ. Press, p. 343

Horstmann H., Schücker P., Seitter W., et al., 1989, Bull. Inf. CDS 37, 43

Kharchenko N.V., Schilbach E., Scholz R.-D., 1994, Astron. Nachr. 315, 291

Kharchenko N.V., Schilbach E., 1995, Astron. Nachr. 316, 91

Kibblewhite E., Bridgeland M., Bunclark P., Cawson M., Irwin M., 1984, Analysis of Images with the APM System at Cambridge. In: Capaccioli M. (ed.) Proc. of Conference on Astronomy with Schmidt-Type Telescopes. Reidel, Dordrecht, p. 89

Kovalevsky J., Lindegren L., Johnston K.J., et al., 1997, A\&A (in press)

Lindegren L., Kovalevsky J., 1995, A\&A 304, 189

Schilbach E., 1984, Dissertation, Potsdam

Schilbach E., 1988, The Tautenburg Part of the Programme studying the Main Meridional Section of the Galaxy. In: Debarbat S. et al. (eds.) Proc. IAU Symp. 133, Mapping the sky. Reidel, Dordrecht, p. 451

Schilbach E., Scholz R.-D., 1991, Astron. Nachr. 312, 377

Schilbach E., Scholz R.-D., 1992, Astron. Nachr. 313, 243

Scholz R.-D., Hirte S., 1991, Astron. Nachr. 312, 45

Scholz R.-D, 1991, Dissertation Abstract, Astron. Nachr. 312, 224

Scholz R.-D, Irwin M.J., 1994, Absolute proper motions of the dwarf spheroidal galaxies in Draco and Ursa Minor. In: MacGillivray H.T. et al. (eds.) Proc. IAU Sympos. 161, Astronomy from wide-field imaging. Kluwer Acad. Publ., Dordrecht, p. 535

Scholz R.-D., Kharchenko N.V., 1994, Astron. Nachr. 315, 73

Scholz R.-D., Odenkirchen M., Irwin M.J., 1994, MNRAS 266, 925

Scholz R.-D., Odenkirchen M., Hirte S., Irwin M.J., Börngen F., Ziener R., 1996, MNRAS 278, 251

Scholz R.-D., Meusinger H., Irwin M.J., 1996, AG Abst. Ser. 12,82

Sergeev A.V., Schornikov O.E., 1984, In Novaja tekhnika v Astronomii, Leningrad, p. 86

Tucholke H.-J., 1995, AG Abst. Ser. 11, 96

Yatsenko A.I., Rybka S.P., Scholz R.-D., 1987, Astron. Nachr. 308,319 